\title{
$\mathbb{N}$ KIT
}

Karlsruhe Institute of Technology

\section{Aortic Coarctation simulation based on the Lattice Boltzmann method: bench- mark results}

T. Henn, V. Heuveline, M. Krause, S. Ritterbusch

No. 2012-06

Preprint Series of the Engineering Mathematics and Computing Lab (EMCL)

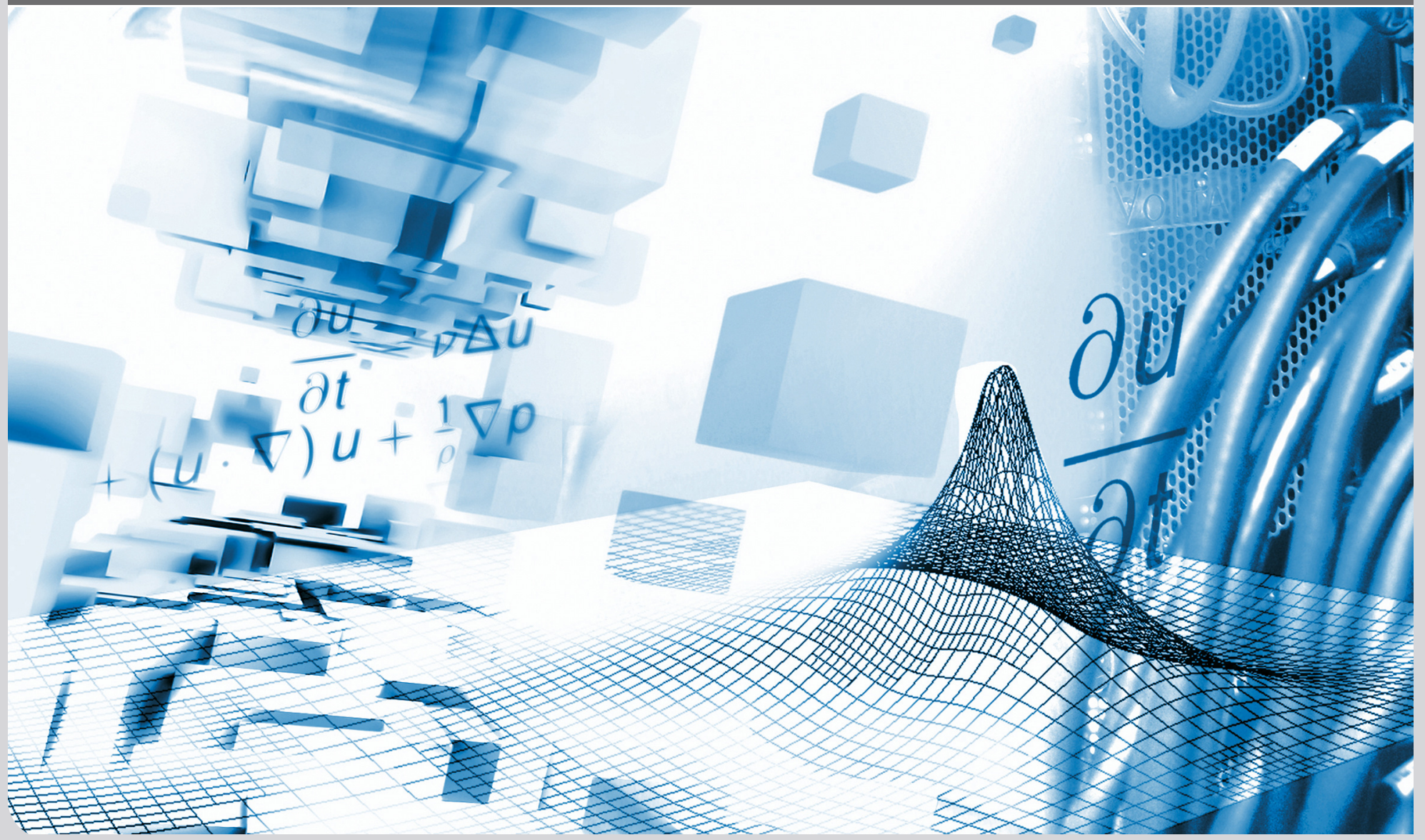

KIT - University of the State of Baden-Wuerttemberg and National Research Center of the Helmholtz Association 
Preprint Series of the Engineering Mathematics and Computing Lab (EMCL) ISSN 2191-0693

No. $2012-06$

\section{Impressum}

Karlsruhe Institute of Technology (KIT)

Engineering Mathematics and Computing Lab (EMCL)

Fritz-Erler-Str. 23, building 01.86

76133 Karlsruhe

Germany

KIT - University of the State of Baden Wuerttemberg and National Laboratory of the Helmholtz Association

Published on the Internet under the following Creative Commons License: http://creativecommons.org/licenses/by-nc-nd/3.0/de .

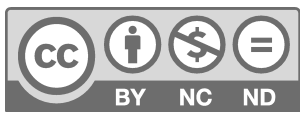




\title{
Aortic Coarctation simulation based on the Lattice Boltzmann method: benchmark results.
}

\author{
Thomas Henn ${ }^{1}$, Vincent Heuveline ${ }^{1}$, Mathias J. Krause ${ }^{2}$, and \\ Sebastian Ritterbusch ${ }^{1}$ \\ ${ }^{1}$ Engineering Mathematics and Computing Lab, \\ Karlsruhe Institute for Technology (KIT), \\ Kaiserstrasse 12, 76131 Karlsruhe, Germany \\ 2 Institute for Algebra and Geometry \\ Karlsruhe Institute of Technology (KIT), \\ Kaiserstr. 93, 76128 Karlsruhe, Germany
}

\begin{abstract}
We investigate a patient specific blood flow simulation through a transverse aortic arch with a moderate thoracic aortic coarctation, where particular attention is paid to the blood pressure gradient through the coarctation. The challenge in this context is the complex geometry containing a stenosis, which results in complex flow patterns. The fluid is assumed to be incompressible and Newtonian. Its dynamic is usually described by an Navier-Stokes equation with appropriate boundary conditions. Instead, we modeled the problem mesoscopically by a family of BGK-Boltzmann equations those solutions reaches that of a corresponding Navier-Stokes system in a certain limit. For discretization we take advantage of lattice Boltzmann methods, which are realized within the open-source library OpenLB. A realistic transient flow profile of the cardiac output for a human at rest was used to specify the inflow boundary condition at the aortic root, whereas the outflow at the descending aorta was modeled by a pressure boundary condition. A short introduction to lattice Boltzmann methods is provided and especially the used boundary conditions are introduced in detail. The exact simulation setup is stated and the obtained results are discussed.
\end{abstract}

\section{Introduction}

Patient-specific numerical simulations of human organs open new opportunities for medical diagnosis and therapy. They are even more advantageous, if they do not require additional radiating screenings, but are based on computer tomography imaging from standard procedures. In the case of the human respiratory system, numerical simulations of air flow have already proven to be accurate in the United Airways project [1]. Therefore, an adaption of the concept to human blood flow is highly appreciated.

An anomaly in the human cardiovascular system, such as a coarctation of the aorta, obstructs the body's supply of nutrients and stresses the heart. In particular, the narrowing can lead to an intense drop in pressure, which directly 
affects the health of the patient. This pathological case accounts approximately one out of ten of all congenital heart defects, and is usually corrected surgically or by use of a catheter.

A conventional measurement of the pressure drop under resting conditions is an easy task for a clinician, but measuring the pressure gradient under exercise conditions is more challenging. Usually artificial stress is created by administering a drug to increase heart rate and contractibility. As this may have unwanted side-effects, it opens up a range of applications for CFD techniques. By simulation of a section of the blood system mimicking the real situation, the health-endangering measurements can be shifted into a virtual model. In this paper we investigate to what extent the lattice Boltzmann method (LBM) is of significance for the present medical case of an eight year old female patient. It is worth mentioning that the used model is likely to be expanded by an elastic model of the aorta geometry to achieve more realistic results.

\section{Modelling}

Let $\Omega \subset \mathbb{R}^{3}$ be a bounded domain representing the aorta, $\left[t_{0}, t_{1}\right]$ a considered time interval and $p: \Omega \times\left[t_{0}, t_{1}\right] \rightarrow \mathbb{R}$ denotes the physical pressure. We denote by $\rho \in \mathbb{R}$ the mass density of the blood which is constant due to the incompressibility of the fluid. And let $\mu \in \mathbb{R}^{3}$ be the viscosity of the blood. The aorta inlet area is denoted by $\Sigma_{\text {in }} \subset \partial \Omega$ and the aorta outlet area is $\Sigma_{\text {out }} \subset \partial \Omega$ which contains the outlet areas of the brachiocephalic artery, left common carotid artery, left subclavian artery and aorta. The outer normal vector on $\partial \Omega$ is denoted by $n$. Shear rates in large arteries are typically sufficiently large to assume that blood behaves as a Newtonian fluid, [2]. Therefore, blood flow in the thoracic aorta and the three major branches is governed by the time dependent Navier-Stokes equations, see below.

Problem 1. Find the fluid velocity $\boldsymbol{u} \in\left[L^{2}\left(\Omega \times\left[t_{0}, t_{1}\right]\right)\right]^{3}$ and the pressure $p \in$ $L^{2}\left(\Omega \times\left[t_{0}, t_{1}\right]\right)$ that fulfill the incompressible Navier-Stokes equations

$$
\begin{aligned}
\frac{\partial \boldsymbol{u}}{\partial t}+(\boldsymbol{u} \cdot \nabla) \boldsymbol{u}-\mu \Delta \boldsymbol{u} & =-\frac{1}{\rho} \nabla p & & \text { in } \Omega \times\left[t_{0}, t_{1}\right], \\
\nabla \boldsymbol{u} & =0 & & \text { in } \Omega \times\left[t_{0}, t_{1}\right],
\end{aligned}
$$

with the appropriate boundary conditions, where $I$ denotes the unity matrix.

Dirichlet condition

$$
\boldsymbol{u}=\boldsymbol{g} \quad \text { on } \Sigma_{\text {in }} \times\left[t_{0}, t_{1}\right]
$$

"do nothing" condition

$$
\begin{aligned}
& (-I p+\mu \nabla \boldsymbol{u}) \cdot \boldsymbol{n}=0 \quad \text { on } \Sigma_{\text {out }} \times\left[t_{0}, t_{1}\right], \\
& \text { no - slip condition }
\end{aligned}
$$

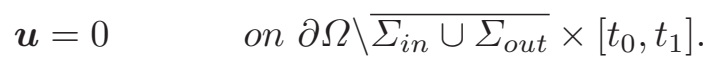


The non-linearity of the problem w.r.t. $\boldsymbol{u}$ comes from the term $(\boldsymbol{u} \cdot \nabla) \boldsymbol{u}$ in the PDE (1). According to [3], [4] in case of stationarity and a sufficiently smooth right hand side there exists at most one strong solution for the velocity $\boldsymbol{u}$, whereas the solution for the pressure is only defined up to an additive constant [5].

\section{Lattice Boltzmann Method}

The subclass of lattice Boltzmann methods considered here enables the simulation of the dynamics of incompressible Newtonian fluids which is usually described macroscopically by an initial value problem governed by a Navier-Stokes equation. Instead of directly computing the quantities of interests, which are the fluid velocity $\boldsymbol{u}=\boldsymbol{u}(t, \boldsymbol{r})$ and fluid pressure $p=p(t, \boldsymbol{r})$ where $\boldsymbol{r} \in \Omega$ and $t \in\left[t_{0}, t_{1}\right] \subseteq \mathbb{R}$, a lattice Boltzmann numerical model simulates the dynamics of particle distribution functions $f:\left[t_{0}, t_{1}\right] \times \Omega \times \mathbb{R}^{3} \rightarrow \mathbb{R}_{>0}$ in a phase space $\Omega \times \mathbb{R}^{3}$ with position $r \in \Omega$ and particle velocity $\boldsymbol{v} \in \mathbb{R}^{3}$. The continuous transient phase space is replaced by a discrete space with a spacing of $\Delta r=h$ for the positions, a set of $q \in \mathbb{N}$ vectors $\boldsymbol{v}_{\boldsymbol{i}} \in \mathcal{O}\left(h^{-1}\right)$ for the velocities and a spacing of $\Delta t=h^{2}$ for time. The resulting discrete phase space is called the lattice and is labeled with the term $D d Q q$. To reflect the discretization of the velocity space, the continuous distribution function $f$ is replaced by a set of $q$ distribution functions $f_{i}:\left[t_{0}, t_{1}\right] \times \Omega \rightarrow \mathbb{R}_{\geq 0}(i=0,1, \ldots, q-1)$, representing an average value of $f$ in the vicinity of the velocity $\boldsymbol{v}_{i}$. Detailed derivations of various LBM can be found in the literature, e.g. in [6]. The iterative process in an LB algorithm can be written in two steps as follows, the collision step (6) and the streaming step (7):

$$
\begin{aligned}
\tilde{f}_{i}(t, \boldsymbol{r}) & =f_{i}(t, \boldsymbol{r})-\frac{1}{3 \nu+1 / 2}\left(f_{i}(t, \boldsymbol{r})-M_{f_{i}}^{e q}(t, \boldsymbol{r})\right), \\
f_{i}\left(t+h^{2}, \boldsymbol{r}+h^{2} \boldsymbol{v}_{i}\right) & =\widetilde{f}_{i}(t, \boldsymbol{r})
\end{aligned}
$$

for $i=0,1, \ldots, q-1$, where

$$
M_{f_{i}}^{e q}(t, \boldsymbol{r}):=\frac{w_{i}}{w} \rho_{f_{i}}\left(1+3 h^{2} \boldsymbol{v}_{i} \cdot \boldsymbol{u}_{f_{i}}-\frac{3}{2} h^{2} \boldsymbol{u}_{f_{i}}^{2}+\frac{9}{2} h^{4}\left(\boldsymbol{v}_{i} \cdot \boldsymbol{u}_{f_{i}}\right)^{2}\right),
$$

is a discretized Maxwell distribution with moments $\rho$ and $\boldsymbol{u}$ which are given according to

$$
\rho:=\sum_{i=0}^{q-1} f_{i} \quad \text { and } \quad \rho \boldsymbol{u}:=\sum_{i=0}^{q-1} \boldsymbol{v}_{i} f_{i} .
$$

Here the variable $\boldsymbol{u}$ is the discrete fluid velocity and $\rho$ the discrete mass density. The kinematic fluid viscosity $\nu=\mu / \rho$ is assumed to be given, and the terms $w_{i} / w, \boldsymbol{v}_{\boldsymbol{i}} h(i=0,1, \ldots, q-1)$ are constants depending on the used lattice Boltzmann method. For more detailed information concerning lattice Boltzmann methods we refer to [7]. The discrete fluid velocity $\boldsymbol{u}$ and the discrete mass density $\rho$ can be related to the solution of a macroscopic initial value problem governed by an incompressible Navier-Stokes equation as shown in [8]. 


\section{Numerical Study}

\subsection{Simulation setup}

A given surface $\partial \Omega$ of an aorta with a moderate thoracic aortic coarctation is voxelized by 5 different resolutions, reaching from $235 \times 118 \times 402$ to $1168 \times 582 \times$ 2002 voxels ( $c f$. Figure 1 ). Recorded data of 20 measurements of the ascending aortic flow is interpolated by cubic splines with periodic boundary conditions. A smooth start-up phase is added to suppress undesired pressure fluctuation. The resulting function is illustrated in Figure 2. A velocity boundary condition, as introduced by Skordos in [9], with a Poiseuille flow profile reflecting the measured flow volume is set at the ascending aortic opening. The blood flow through the upper branch vessels was experimentally measured as a percentage of the ascending aortic flow. Therefore the flow through the left carotid artery is set to be $11.3 \%$ of the aortic flow, whereas the flow through the left subclavian artery is set to be $4.26 \%$. For the right carotid and the right subclavian only a combined value of $25.6 \%$ was available. Hence the flow through these two arteries was calculated depending on the areas of the openings and chosen to be $10.9 \%$ and $14.7 \%$, respectively. The boundary conditions at the descending aorta is set as pressure condition, i.e. the pressure is fixed to $0 \mathrm{mmHg}$ for all times. A full-way bounce back condition is assigned to the remaining surface. The blood is assumed to be Newtonian with a density of $\rho=0.001 \mathrm{gr} / \mathrm{mm}^{3}$ and a dynamic viscosity of $\mu=0.004 \mathrm{gr} / \mathrm{mm} / \mathrm{s}$. A D3Q19 BGK-Lattice Boltzmann Method, supported by a Smagorinsky turbulence model with constant $C_{S}=0.12$, was used. The simulations were executed using the open-source library OpenLB ${ }^{3}$. Computation times vary between approx. 1.5 hours on 64 Intel Xeon X5650@2.67GHz cores for the smallest resolution and 6 days occupying 512 AMD Opteron@2.6GHz cores for the highest resolution.

\subsection{Results}

Two cardiac cycles have been simulated and the pressure drop at the aortic coarctation was determined by calculation of the spatial pressure average in two planes $\pi_{1}$ and $\pi_{2}$ (cf. Figure 1$)$. The absolute pressure over time at the ascending aorta and the pressure drop around the coarctation are shown in Figures 3 and 4 for different spatial resolutions. It is found that with increasing resolution the resulting curves become less smooth. This effect is more pronounced in the graphs of the pressure drop and may be due to the fact that with increasing resolution small turbulence become more significant, which otherwise are smoothed.

Figure 1 shows streamlines representing the flow and velocity $\boldsymbol{u}$ at the time of highest inflow. As expected the velocity increases in the coarctation. The flow is predominantly laminar except for turbulence in the areas before and after the coarctation.

In Table 1 the peak and mean pressure difference around the coarctation, the flow splits through the upper arteries and the descending aorta as a percentage

\footnotetext{
${ }^{3}$ http://www.openlb.org
} 

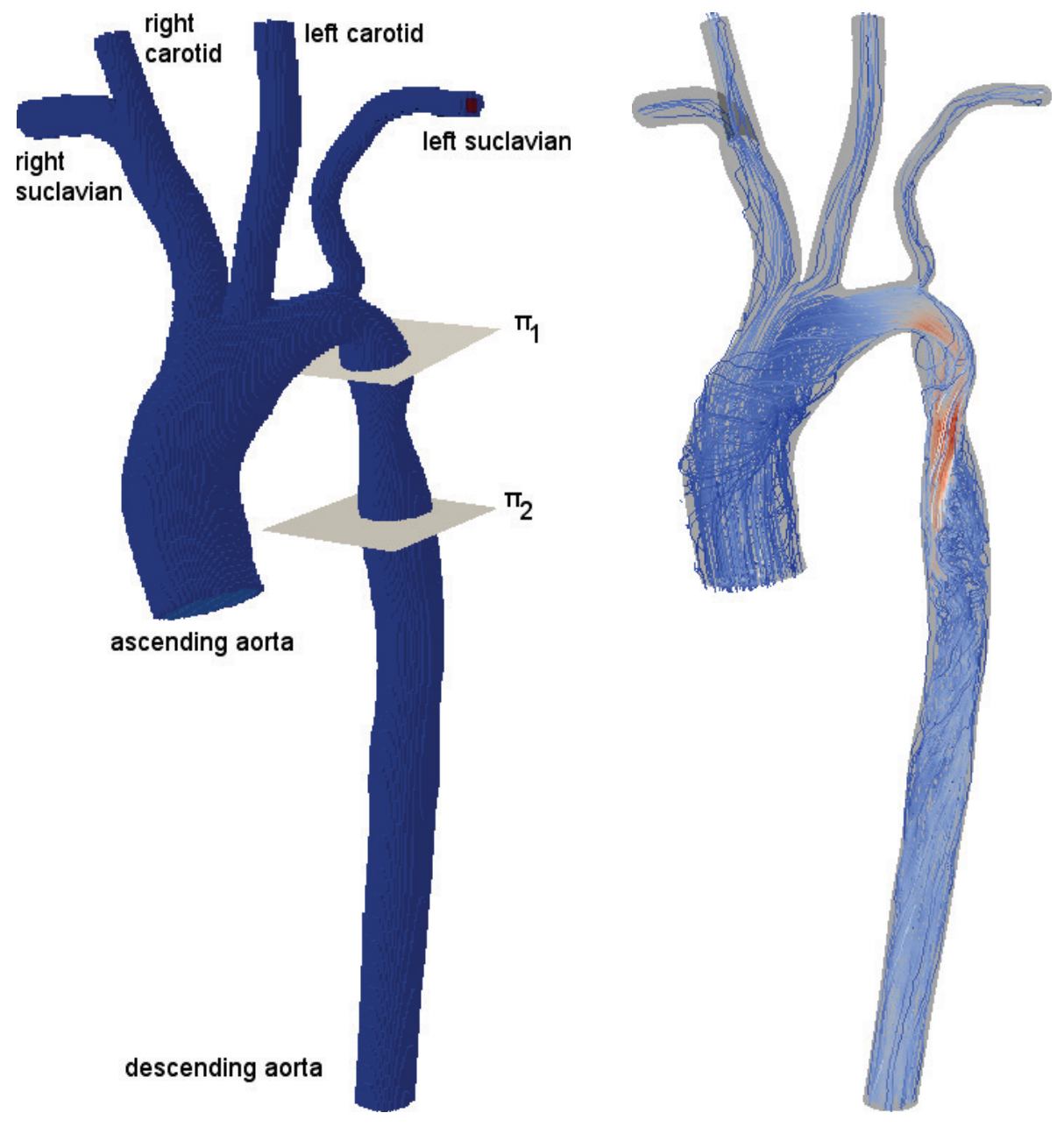

Fig. 1: Voxelized geometry $\Omega_{h}$ of the aortic arc with spacial resolution of $235 \times$ $118 \times 402$ voxels and flow visualizations at the point of time of highest flow rate. Color indicates the flow velocity $\|\boldsymbol{u}\|$.

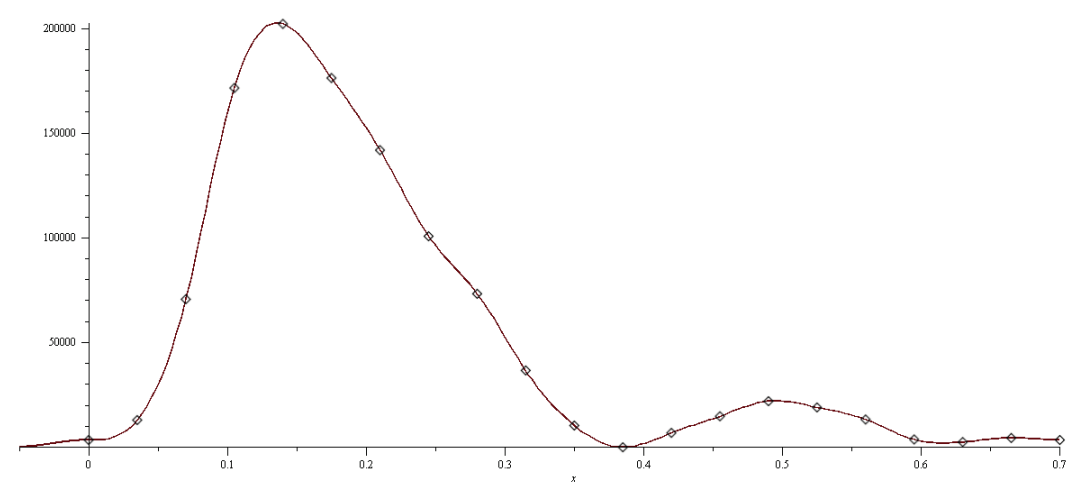

Fig. 2: Cubic spline interpolation of the provided flow information at the ascending aorta $\left[\mathrm{ml} \mathrm{s}^{-1} / \mathrm{s}\right]$. The marked points represent the measured data. 
of the flow through the ascending aorta and systolic and diastolic pressure in the ascending aorta are listed for all simulations. As the pressure is only determined up to an additive constant when solving the Navier-Stokes equations in our context, the values of the absolute pressure at the ascending aorta have been shifted to fit the measured systolic and diastolic pressure of $115 \mathrm{mmHg}$ and $65 \mathrm{mmHg}$. It is found that the pressure is decreasing with increasing spatial and time discretization.

Assuming the measured systolic pressure of $115 \mathrm{mmHg}$ as solution, we obtain the experimental order of convergence (EOC) for $0<h_{1}<h_{2}$, which is defined by

$$
\operatorname{EOC}\left(h_{1}, h_{2}\right):=\frac{\ln \left(e r r_{h 1} / e r r_{h 2}\right)}{\ln \left(h_{1} / h_{2}\right)},
$$

where $\operatorname{err}_{h}=p_{h}^{\text {sys }}-115$ is the error of the computed systolic pressure with respect to the measured value for a given spacing $h$.

\begin{tabular}{l|l|l}
$h_{2}$ & $h_{1}$ & EOC \\
\hline \hline $1 / 402$ & $1 / 802$ & 1.413 \\
\hline $1 / 802$ & $1 / 1202$ & 1.436 \\
\hline $1 / 1202$ & $1 / 1602$ & 1.261 \\
\hline $1 / 1602$ & $1 / 2002$ & 1.008
\end{tabular}

From this table we see that this lattice Boltzmann method yields a systolic pressure $p_{h}^{\text {sys }}$ of linear order. For the highest spatial and time resolution we obtain $p_{h}^{\text {sys }}=116.97 \mathrm{mmHg}$.

\section{Conclusion}

In this paper, we presented how lattice Boltzmann methods can be used for hemodynamic simulations in an aortic model. Here, a discrete Boltzmann equation is solved to simulate a Newtonian fluid on a mesoscopic scale. Since the numerical solution depends on the spatial discretization (note that the temporal resolution is coupled to the spatial resolution $c f$. Section 3), we have experimentally found a linear convergence of the solution $p$ in $h$. The numerical investigations have shown that this approach may well be used for patient-specific simulations, because for high temporal and spatial resolution of the solution space, we obtain realistic values for the pressure (see right column of Table 1). These results could be further improved by extension of the underlying model, e.g. by a Windkessel approach.

\subsection{Acknowledgment}

This work is supported by the German "Federal Ministry of Education and Research (BMBF)": BMBF-Fördermassnahme KMU-innovativ: Informations- und 

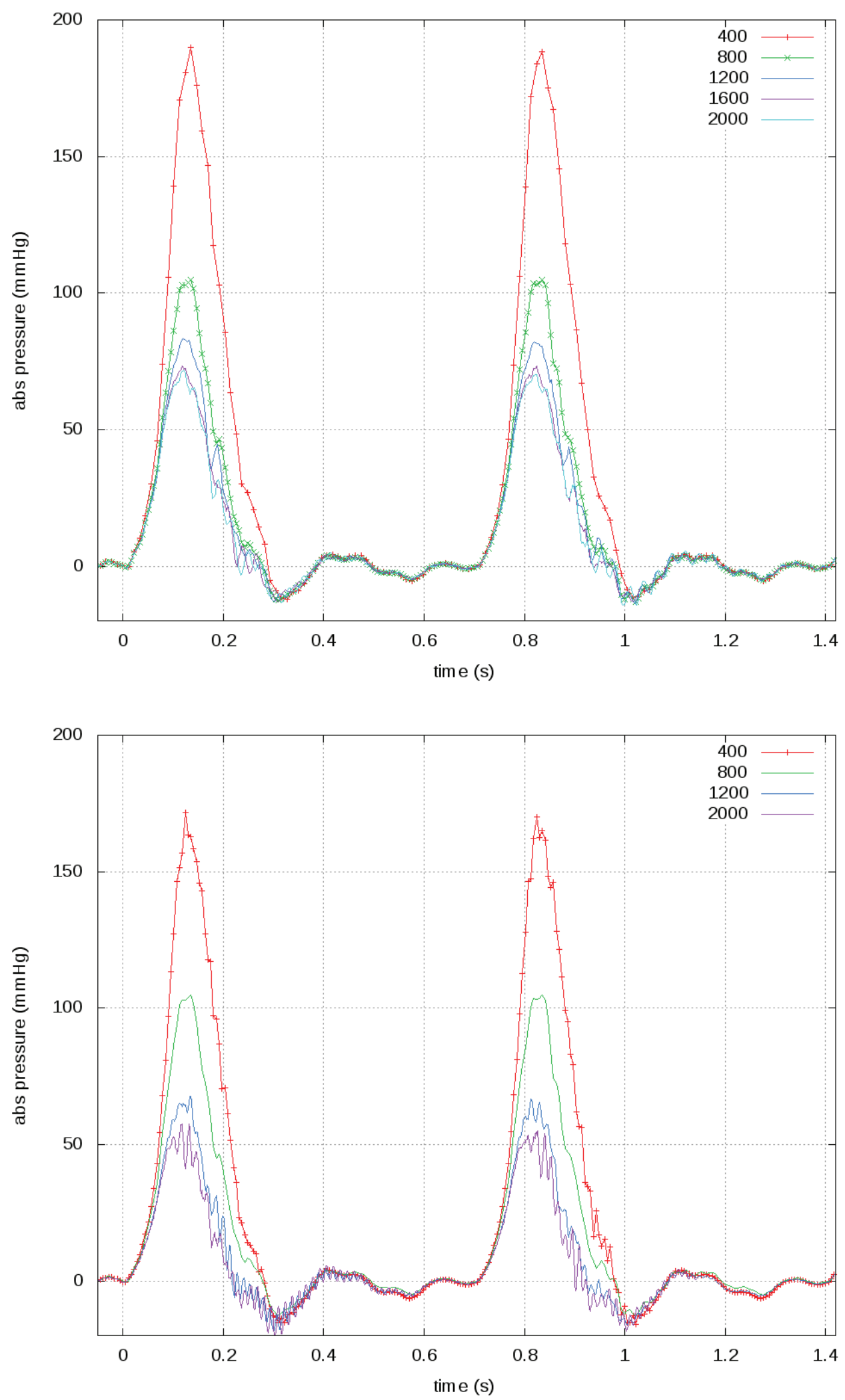

Fig. 3: The absolute pressure over time at the ascending aorta is shown for different spacial resolutions. The upper graph shows the resulting curve for the higher time resolution. The according time-step sizes can be found in Table 1 . 

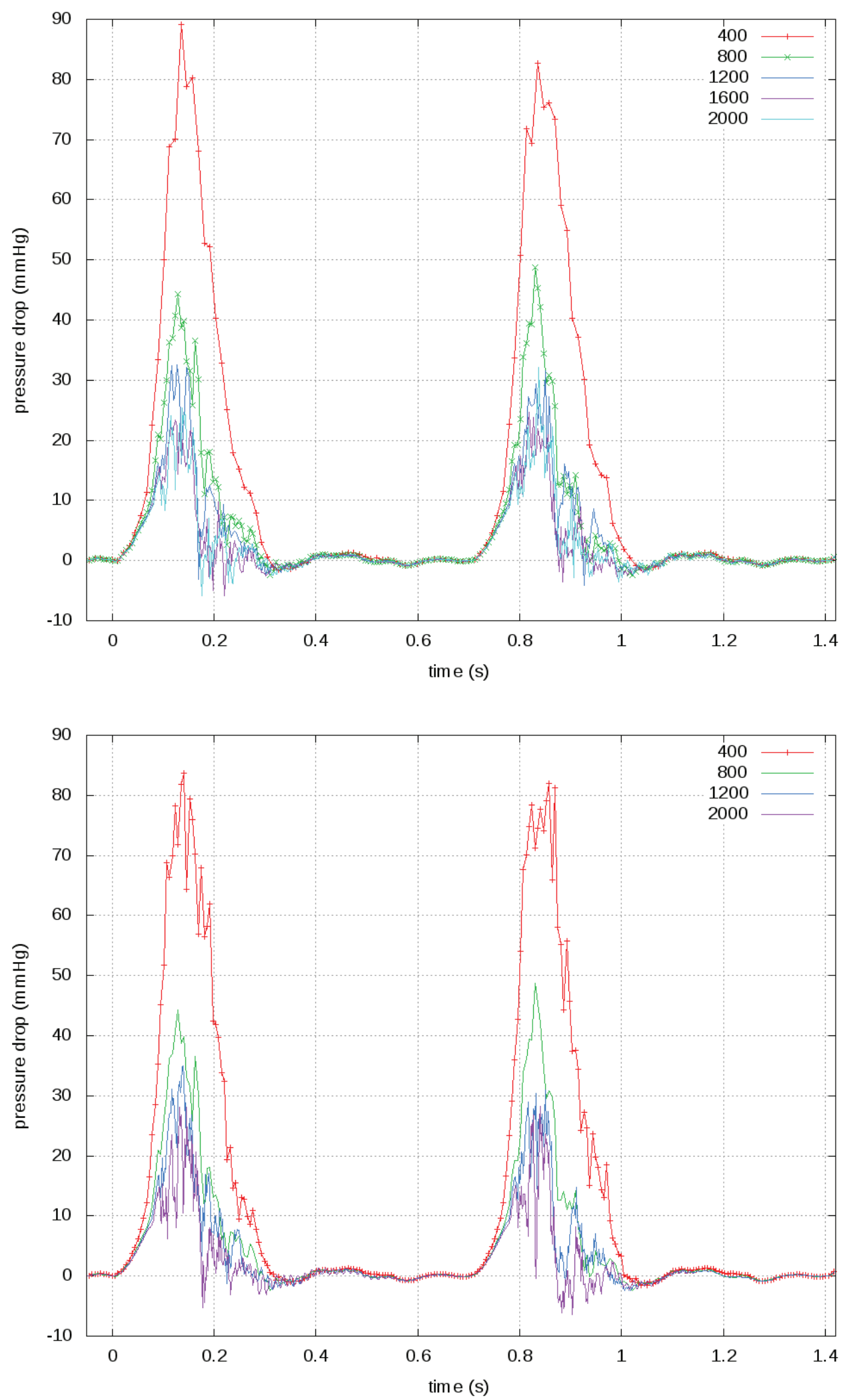

Fig. 4: The pressure drop between the planes $\pi_{1}$ and $\pi_{2}$ around the coarctation are shown for different spatial resolutions. The upper graph shows the resulting curve for the higher time resolution. The according time-step sizes can be found in Table 1. 


\begin{tabular}{|c|c|c|c|c|c|c|c|c|c|c|c|c|}
\hline & $\begin{array}{l}0 \\
\stackrel{0}{\circ} \\
\text { t. }\end{array}$ & 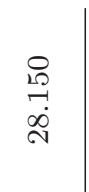 & $\begin{array}{l}\mathscr{D} \\
\stackrel{0}{ } \\
\stackrel{i}{i}\end{array}$ & 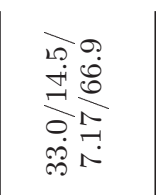 & 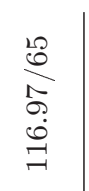 & $\begin{array}{l}\vec{N} \\
\text { ָे } \\
\stackrel{\sim}{N}\end{array}$ & & & 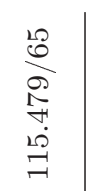 & \multirow{3}{*}{ 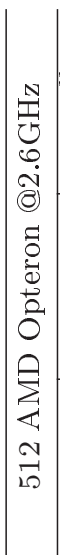 } & 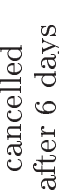 & \multirow{10}{*}{ 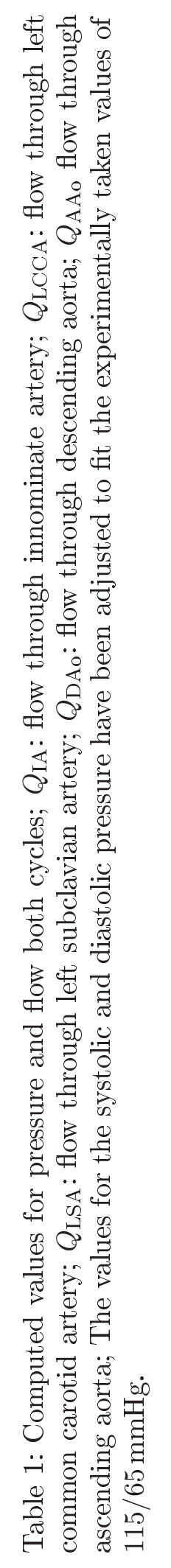 } \\
\hline & $\begin{array}{l}1 \\
\stackrel{0}{\circ} \\
\stackrel{\leftrightarrow}{\rightarrow}\end{array}$ & 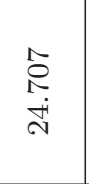 & 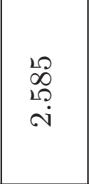 & 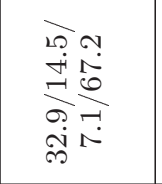 & 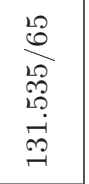 & 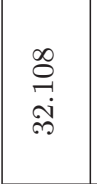 & $\begin{array}{l}\infty \\
\infty \\
\stackrel{1}{i} \\
\text { in }\end{array}$ & 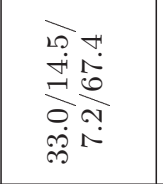 & 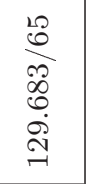 & & $\begin{array}{l}\vec{\infty} \\
\stackrel{0}{0} \\
\stackrel{2}{L} \\
\tilde{0} \\
-1\end{array}$ & \\
\hline 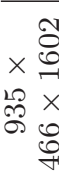 & 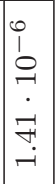 & 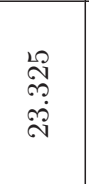 & $\begin{array}{l}0 \\
\stackrel{0}{0} \\
\stackrel{\circ}{i} \\
\text { in }\end{array}$ & 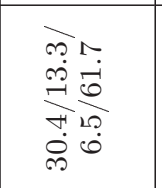 & 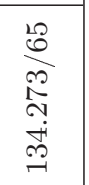 & 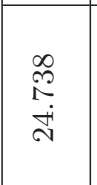 & 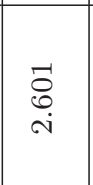 & 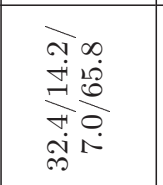 & 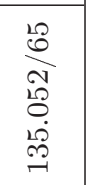 & & 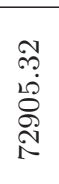 & \\
\hline 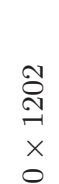 & 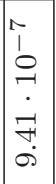 & $\begin{array}{l}\overrightarrow{0} \\
0 \\
\dot{\phi}\end{array}$ & $\begin{array}{l}\vec{D} \\
\infty \\
\text { id }\end{array}$ & 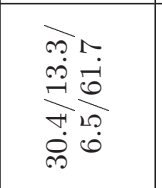 & 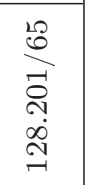 & 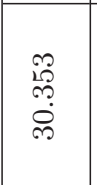 & $\begin{array}{l}\infty \\
0 \\
\llcorner \\
\infty \\
\text { on }\end{array}$ & 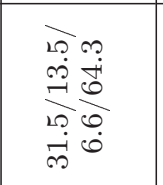 & 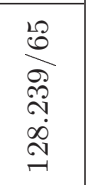 & 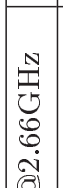 & $\begin{array}{l}\stackrel{0}{1} \\
\vec{D} \\
\infty \\
\infty \\
\infty \\
-1\end{array}$ & \\
\hline 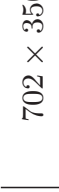 & $\begin{array}{l}0 \\
1 \\
0 \\
0 \\
\dot{\infty} \\
\infty \\
\infty \\
-1 \\
\end{array}$ & $\begin{array}{l}\stackrel{9}{\overrightarrow{1}} \\
\stackrel{10}{0} \\
\stackrel{0}{0}\end{array}$ & $\begin{array}{l}1 \\
\infty \\
\infty \\
\infty \\
\dot{\infty}\end{array}$ & 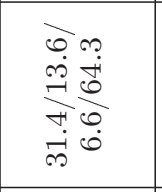 & 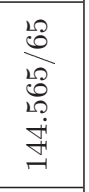 & $\begin{array}{l}\stackrel{8}{ఫ} \\
\stackrel{+}{+}\end{array}$ & $\begin{array}{l}\infty \\
\stackrel{\infty}{\circ} \\
+\end{array}$ & 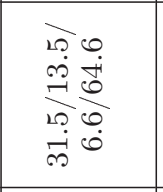 & 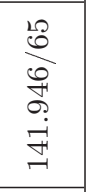 & 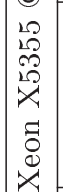 & \begin{tabular}{l}
10 \\
10 \\
$\infty$ \\
\multirow{2}{\$}{} \\
$\overparen{H}$ \\
$\sigma$
\end{tabular} & \\
\hline $\begin{array}{l}\tilde{\omega} \\
\infty \\
\times \\
+\end{array}$ & 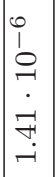 & 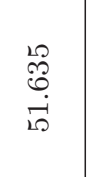 & $\underset{i \infty}{\stackrel{M}{\Gamma}}$ & 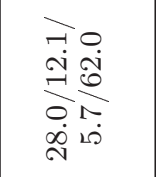 & 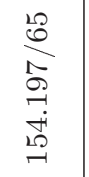 & $\begin{array}{l}\text { Ñ } \\
\text { İ } \\
\stackrel{0}{0}\end{array}$ & $\begin{array}{l}\stackrel{9}{H} \\
\stackrel{\leftrightarrow}{0}\end{array}$ & 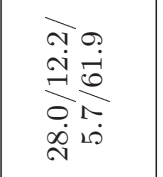 & 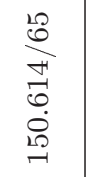 & 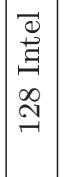 & 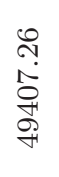 & \\
\hline 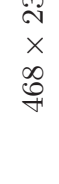 & $\begin{array}{l}0 \\
i \\
o \\
\ddots \\
\dot{\infty} \\
\infty \\
\sim \\
i \\
i\end{array}$ & $\begin{array}{l}\stackrel{0}{L} \\
\stackrel{D}{N} \\
\stackrel{F}{\forall}\end{array}$ & $\begin{array}{l}\infty \\
\infty \\
\infty \\
\qquad\end{array}$ & 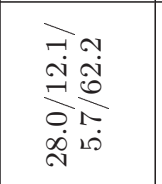 & $\begin{array}{l}\qquad 8 \\
0 \\
0 \\
0 \\
0 \\
0 \\
0 \\
0\end{array}$ & $\begin{array}{l}\overrightarrow{0} \\
\infty \\
\infty \\
\infty \\
o\end{array}$ & 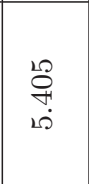 & 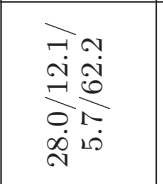 & 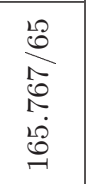 & \multirow{3}{*}{ 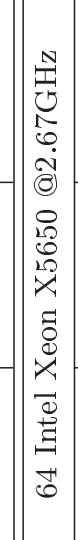 } & 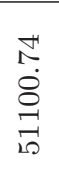 & \\
\hline 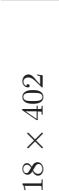 & \begin{tabular}{|l|}
0 \\
1 \\
0 \\
$\stackrel{1}{1}$ \\
$\dot{0}$ \\
$\sim$ \\
$i$ \\
$i$ \\
\end{tabular} & $\begin{array}{l}\stackrel{\mathscr{H}}{\mathcal{H}} \\
\ddot{\infty}\end{array}$ & 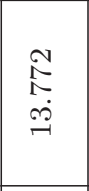 & 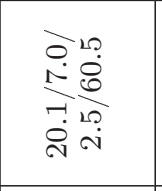 & 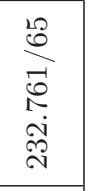 & 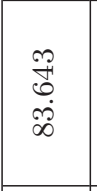 & $\begin{array}{l}\stackrel{\Omega}{\rightrightarrows} \\
\underset{+}{\leftrightarrows}\end{array}$ & 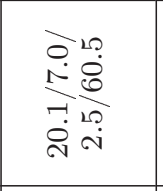 & 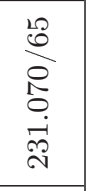 & & 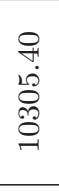 & \\
\hline $\begin{array}{l}\overrightarrow{1} \\
\times \\
100 \\
\stackrel{0}{N}\end{array}$ & $\mid \begin{array}{l}0 \\
1 \\
0 \\
\ddots \\
\dot{j} \\
0 \\
10 \\
10\end{array}$ & 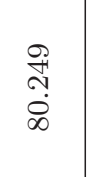 & $\begin{array}{l}\vec{D} \\
0 \\
\stackrel{\vec{g}}{\rightarrow}\end{array}$ & 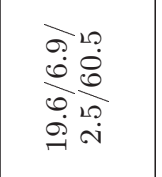 & 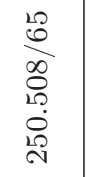 & 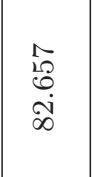 & $\begin{array}{l}\underset{\mathcal{N}}{\mathrm{N}} \\
\stackrel{+}{-}\end{array}$ & 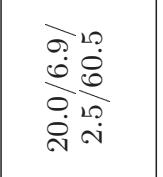 & 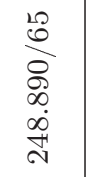 & & 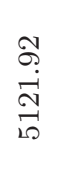 & \\
\hline 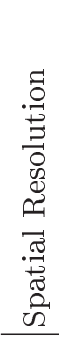 & 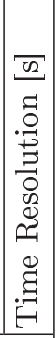 & 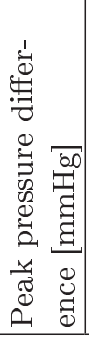 & 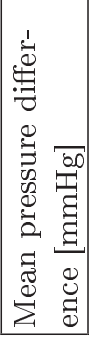 & 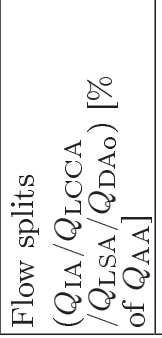 & 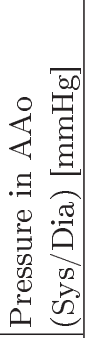 & 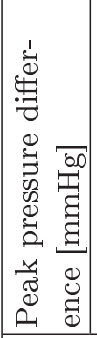 & 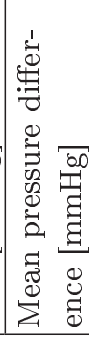 & 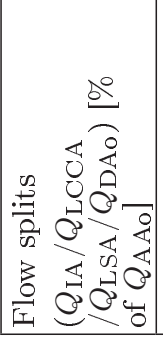 & 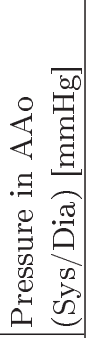 & 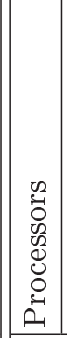 & 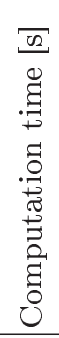 & \\
\hline
\end{tabular}


Kommunikationstechnologie, Forschungsbereich Softwaresysteme und Wissensverarbeitung, Gesundheit und Medizintechnik. Support code 01|S11031B.

We would like to express thanks to our colleague Dr. Andreas HelfrichSchkarbanenko for the numerous discussions and careful proof reading.

\section{References}

1. Krause, M.J., Gengenbach, T., Mayer, R., Zimney, S., Heuveline, V.: How to Breathe Life into CT-Data. Computer Aided Medical Engineering (4) (2011) 29-33

2. Fung, Y.C.: Biomechanics : mechanical properties of living tissues. Springer, New York, NY (1981)

3. Sohr, H.: The Navier-Stokes Equations. Birkhäuser Basel (2001)

4. Temam, R.: Navier-Stokes Equations and Nonlinear Functional Analysis (CBMSNSF Regional Conference Series in Applied Mathematics). Society for Industrial and Applied Mathematics (1987)

5. Marion, M., Temam, R.: Navier-stokes equations: Theory and approximation. In Ciarlet, P., Lions, J., eds.: Numerical Methods for Solids (Part 3) Numerical Methods for Fluids (Part 1). Volume 6 of Handbook of Numerical Analysis. Elsevier (1998) 503-689

6. Sukop, M.C., Thorne, D.T.: Lattice Boltzmann Modeling: An Introduction for Geoscientists and Engineers. Springer (2010)

7. Krause, M.J.: Fluid Flow Simulation and Optimisation with Lattice Boltzmann Methods on High Performance Computers: Application to the Human Respiratory System. PhD thesis, Karlsruhe Institute of Technology (KIT), Universität Karlsruhe (TH), Kaiserstraße 12, 76131 Karlsruhe, Germany (July 2010) http://digbib.ubka.uni-karlsruhe.de/volltexte/1000019768.

8. Junk, M., Klar, A.: Discretizations for the incompressible navier-stokes equations based on the lattice boltzmann method. SIAM Journal on Scientific Computing 22(1) (2000) 1-19

9. Skordos, P.A.: Initial and boundary conditions for the lattice boltzmann method. Phys. Rev. E 48 (Dec 1993) 4823-4842 


\section{Preprint Series of the Engineering Mathematics and Computing Lab}

recent issues

No. 2012-05 Vincent Heuveline, Eva Ketelaer, Staffan Ronnas, Mareike Schmidtobreick, Martin Wlotzka: Scalability Study of HiFlow ${ }^{3}$ based on a Fluid Flow Channel Benchmark

No. 2012-04 Hartwig Anzt, Armen Beglarian, Suren Chilingaryan, Andrew Ferrone, Vincent Heuveline, Andreas Kopmann: A unified Energy Footprint for Simulation Software

No. 2012-03 Vincent Heuveline, Chandramowli Subramanian: The Coffee-table Book of Pseudospectra

No. 2012-02 Dominik P.J. Barz, Hendryk Bockelmann, Vincent Heuveline: Electrokinetic optimization of a micromixer for lab-on-chip applications

No. 2012-01 Sven Janko, Björn Rocker, Martin Schindewolf, Vincent Heuveline, Wolfgang Karl: Software Transactional Memory, OpenMP and Pthread implementations of the Conjugate Gradients Method - a Preliminary Evaluation

No. 2011-17 Hartwig Anzt, Jack Dongarra, Vincent Heuveline, Piotr Luszczek: GPU-Accelerated Asynchronous Error Correction for Mixed Precision Iterative Refinement

No. 2011-16 Vincent Heuveline, Sebastian Ritterbusch, Staffan Ronnås: Augmented Reality for Urban Simulation Visualization

No. 2011-15 Hartwig Anzt, Jack Dongarra, Mark Gates, Stanimire Tomov: Block-asynchronous multigrid smoothers for GPU-accelerated systems

No. 2011-14 Hartwig Anzt, Jack Dongarra, Vincent Heuveline, Stanimire Tomov: A Block-Asynchronous Relaxation Method for Graphics Processing Units

No. 2011-13 Vincent Heuveline, Wolfgang Karl, Fabian Nowak, Mareike Schmidtobreick, Florian Wilhelm: Employing a High-Level Language for Porting Numerical Applications to Reconfigurable Hardware

No. 2011-12 Vincent Heuveline, Gudrun Thäter: Proceedings of the 4th EMCL-Workshop Numerical Simulation, Optimization and High Performance Computing

No. 2011-11 Thomas Gengenbach, Vincent Heuveline, Mathias J. Krause: Numerical Simulation of the Human Lung: A Two-scale Approach

No. 2011-10 Vincent Heuveline, Dimitar Lukarski, Fabian Oboril, Mehdi B. Tahoori, Jan-Philipp Weiss: Numerical Defect Correction as an Algorithm-Based Fault Tolerance Technique for Iterative Solvers

No. 2011-09 Vincent Heuveline, Dimitar Lukarski, Nico Trost, Jan-Philipp Weiss: Parallel Smoothers for Matrix-based Multigrid Methods on Unstructured Meshes Using Multicore CPUs and GPUs

No. 2011-08 Vincent Heuveline, Dimitar Lukarski, Jan-Philipp Weiss: Enhanced Parallel ILU $(p)$-based Preconditioners for Multi-core CPUs and GPUs - The Power $(q)$-pattern Method 